\title{
Research on Oil-immersed Transformer Fault Diagnosis Method Based on Improved Extension Set on Intervals
}

\author{
Zhou Huayanran
}

\author{
North China Electric Power University 071006
}

\begin{abstract}
Key words: transformer fault diagnosis, improved analytic hierarchy process, extension set on intervals, maximum subordination principle
\end{abstract}

\begin{abstract}
To solve the following problems during Transformer Fault Diagnosis: high misdiagnosis rate, calculation complexity, low recognition rate and so on, Oil-immersed Power Transformer fault diagnosis method based on Improved Interval Extension is put forward. First, Improved Extension Set on Intervals fault diagnosis model is established by interval extension method; then, the improved analytic hierarchy process is used to calculate and select the fault feature weight value of typical gases in oil, and the classical and joint domain are obtained according to fault feature standard sequence of typical gases in oil. Finally, the fault type of the object to be diagnosed can be obtained by relevancy calculation and the maximum subordination principle. Then, a test based on 30 sets of data from each fault type is carried out, the result of which verify the effectiveness of the method proposed.
\end{abstract}

\section{Introduction}

With the advent of a new era of the power system in which the smart grid and ultra-high voltage emerge, the power supply nowadays has grown a lot in importance. Since the power transformer is an important part of the power system ${ }^{[1-2]}$, the research on its fault diagnosis is of great significance. At present, the frequently-adopted transformer fault diagnosis method is dissolved gas analysis, which mainly involves the total hydrocarbon ann $\mathrm{v}$ curve method, four ratio method, artificial neutral network technology and three ratio method. In this paper, a research on oil-immersed transformer fault diagnosis method based on improved extension set on intervals has been done. In other words, a diagnosis method with the help of the extension theory to recognize the extension in different fault situations of transformer has been put forward. Specifically speaking, the matter element, the classical and joint domain of the diagnosed object will be determined through the interval extension method, and the fault feature weight value of the selected typical gases in oil will be calculated through the improved analytic hierarchy method followed by a calculation of relevancy. Finally, the fault type of the object to be diagnosed can be determined based on the maximum subordination principle. Through this method, both the efficiency and accuracy of the fault diagnosis can be improved.

Analysis on transformer diagnosis and its features. The common three faults of oil-immersed transformer are overheat fault, discharge fault and moisture.In this paper, a rough statistics on the faults of a oil-immersed transformer in Beijing has been done, the result of which has been shown in Table 1. 
Tab.1 Oil-immersed transformer fault statistics

\begin{tabular}{|l|l|l|}
\hline Fault Type & Number & Proportion \\
\hline Overheat Fault & 225 & 53 \\
\hline Arc Discharge Fault & 63 & 18.1 \\
\hline $\begin{array}{l}\text { Overheating Arc Discharge } \\
\text { Fault }\end{array}$ & 38 & 10 \\
\hline Partial Discharge Fault & 24 & 7 \\
\hline $\begin{array}{l}\text { Moisture or Spark } \\
\text { Discharge Fault }\end{array}$ & 8 & 1.9 \\
\hline
\end{tabular}

In this paper, the faults of transformer has been divided into seven categories: low temperature overheat fault, high temperature overheat fault, low energy discharge fault, high energy discharge fault, tree discharge fault, interturn discharge fault, partial discharge fault caused by moisture.

As is indicated in the dissolved gas analysis of transformer, the characteristic gases in oil of the transformer mainly include $\mathrm{H}_{2}, \mathrm{CO}, \mathrm{CO}_{2}$ and a large number of hydrocarbons such as $\mathrm{CH}_{4}, \mathrm{C}_{2} \mathrm{H}_{2}$ and $\mathrm{CH}_{6}$. the following five gases, namely, $\mathrm{H}_{2}, \mathrm{CH}_{4}, \mathrm{C}_{2} \mathrm{H}_{6}, \mathrm{C}_{2} \mathrm{H}_{4}, \mathrm{C}_{2} \mathrm{H}_{2}$, have been selected as the characteristic index quantity of the fault diagnosis in this paper.

\section{Fault Diagnosis Mode Based On Improved Extension On intervals}

(1) Determining the Matter Element

Supposing that there are $\mathrm{n}$ characteristic quantities in the transformer fault, which are named as $\mathrm{C}_{1}, \mathrm{C}_{2}, \mathrm{C}_{3}, \ldots, \mathrm{C}_{\mathrm{n}}$, the matter element mode of the diagnosed object can be determined through the following Formula (1), base do the definition of the extension of matter element.

$$
R=\left(U, C_{k}, v_{k}\right)=\left[\begin{array}{ccc}
U & C_{1} & v_{1} \\
& C_{2} & v_{2} \\
& \vdots & \vdots \\
& C_{n} & v_{n}
\end{array}\right]
$$

In Formula (1), $\mathrm{U}$ refers to the fault type of the diagnosed object, and $\mathrm{V}_{\mathrm{k}}$ refers to the interval of the $\mathrm{K}^{\text {th }}$ characteristic quanlity of the diagnosed object.

(2) Determining the Classical Domain

Supposing there are altogether $m$ types of transformer fault $(j=1,2,3, \ldots, m)$, the classical domain of each fault type can be determined based on its data sequence, which is shown as Formula (2).

$$
R_{j}=\left(U_{j}, C_{k}, v_{k}\right)=\left[\begin{array}{ccc}
U & C_{1} & \left\langle a_{j 1}, b_{j 1}\right\rangle \\
& C_{2} & \left\langle a_{j 2}, b_{j 2}\right\rangle \\
\vdots & \vdots \\
& C_{n} & \left\langle a_{j n}, b_{j n}\right\rangle
\end{array}\right]
$$

In Formula (2), $R_{j}$ refers to the matter element mode of the $j^{\text {th }}$ fault type; $U_{j}$ refers to the diagnosis result of the object with the $j^{\text {th }}$ fault type; $v_{k}=<a_{j k}, b_{j k}>$ refers to the interval of the $k^{\text {th }}$ characteristic quantity when the fault type is $\mathrm{j}$.

(3) Determining the Joint Domain

According to the definition of joint domain, the process of determining the joint domain is one to determine the value-taking interval based on the value-taking scope of the fault characteristic quantity, as is shown in Formula (3). 


$$
R_{p}=\left(U_{p}, C_{k}, v_{p k}\right)=\left[\begin{array}{ccc}
U & C_{1} & v_{p 1} \\
& C_{2} & v_{p 2} \\
\vdots & \vdots \\
& C_{n} & v_{p n}
\end{array}\right]
$$

In Formula (3), $R_{p}$ refers to the joint domain in the matter element mode; $U_{p}$ refers to the transformer fault type; $v_{\mathrm{pk}}$ refers to the value-taking range of the characteristic quantity $\mathrm{C}_{\mathrm{k}}$.

(4) Calculating the Relevancy Based on the Establishment of Relevancy Function

The establishment of relevancy function can make the calculation of relevancy between the object to be diagnosed and the classical and joint domain of the matter element mode more accurate, which doesn't rely on the subjective judgment or statistics ${ }^{[3]}$. The calculation formula of the relevancy between the matter element mode and its classical and joint domain is shown as Formula (4).

$$
\left\{\begin{array}{c}
\rho\left(v_{k}, v_{j k}\right)=\left|v_{k}-\frac{a_{j k}+b_{j k}}{2}\right|-\frac{b_{j k}-a_{j k}}{2} \\
\rho\left(v_{k}, v_{p k}\right)=\left|v_{k}-\frac{a_{p k}+b_{p k}}{2}\right|-\frac{b_{p k}-a_{p k}}{2} \\
\quad(k=1,2,3 \cdots n, j=1,2, \cdots m)
\end{array}\right.
$$

In Formula (4), $\rho$ refers to the proximity between $v_{k}$ and $v_{p k}$. If the value of $\rho$ is more than or equals to zero, a conclusion can be drawn that $v_{k}$ is beyond the interval of $v_{p k}$. If the value of $\rho$ is less than or equals to zero, a conclusion can be drawn that $\mathrm{v}_{\mathrm{k}}$ is within the interval of $\mathrm{v}_{\mathrm{pk}}$, and the value indicates different degrees of closeness.

In addition, the relevancy between $\mathrm{C}_{\mathrm{k}}$-the $\mathrm{k}^{\text {th }}$ matter element index of the diagnosed object and the $\mathrm{j}^{\text {th }}$ fault type can be calculated through the following formula.

$$
K_{j}\left(v_{k}\right)=\frac{\rho\left(v_{k}, v_{j k}\right)}{\rho\left(v_{k}, v_{p k}\right)-\rho\left(v_{k}, v_{j k}\right)}
$$

\section{(5) Weight Determination}

The traditional analytic hierarchy process requires a consistency check, whose calculation is very large. Since the accuracy and efficiency should both be taken into consideration in the actual assessment calculation process, the author of this paper has put forward an improved analytic hierarchy process to check the constancy of the expert assessment, which involves an initial judgment of discrepancy through standard deviation and the establishment of an improved matrix. The specific steps of the improved analytic hierarchy process are as follows:

1) Establishing a Judgment Matrix

Based on the weight assessment set of the determined objects, the nine scaling method is adopted in this paper to describe the relative importance of different characteristic quantities under the same hierarchy. Then the following judgment matrix A which is corresponding to the related index set can be obtained.

2) Calculating the Weight Coefficient by Establishing an Improved Matrix As a judgment matrix, Matrix A is about to go through a consistency check which, as has been mentioned above, is very large in its calculation amount. Therefore, to enhance the calculation efficiency, something must be done to leave out the consistency check. Since the judgment matrix produced by the analytic hierarchy process is reciprocal, the suggested way to do that in this paper is to convert Matrix A-a reciprocal one into Matrix $\mathrm{H}$-an antisymmetric one though the formula 
$\mathrm{H}=\operatorname{lgA}$. In this way, all the elements in the matrix become integers with an absolute value larger than or equivalent to one. Besides, since all the elements in Matrix $\mathrm{H}$ have such a relationship: $\mathrm{h}_{\mathrm{ij}}=\mathrm{h}_{\mathrm{ik}}+\mathrm{h}_{\mathrm{k}}$, and Matrix $\mathrm{H}$ is transmissible, the element $\mathrm{c}_{\mathrm{ij}}$ in Matrix D-a transfer one of Matrix $\mathrm{H}$ can be described by Formula (6).

$$
\varepsilon=\sum_{i=1}^{n} \sum_{j=1}^{n}\left(d_{i j}-h_{i j}\right)^{2}
$$

When $\varepsilon=\varepsilon_{\text {min }}$, Matrix D, an optimal transfer matrix of Matrix H, can be obtained. If $10^{\mathrm{D}}$ is assigned to $A_{\mathrm{r}}$ as its value, $A_{\mathrm{r}}$ meets the requirement of consistency. In this way, what one needs to do is to work out the characteristic vector quantity instead of the consistency check.

3) Multilevel Extension Evaluation

Based on the calculated weight, the relevancy between the object to be recognized and each fault type can be worked out through Formula (7). And then the fault type of the transformer can be determined according to the maximum subordination principle, as is shown in Formula (8).

$$
\begin{aligned}
K_{i}(U) & =\sum_{i=1}^{n} w_{i} K_{j} \\
& K_{j}(U)=\max _{1 \leq j \leq m} K_{j}(U)
\end{aligned}
$$

\section{An Instance Analysis of the Transformer Fault Diagnosis Based on Improved Extension on intervals}

According to the fault diagnosis modes based on the interval extension method, $\mathrm{C}_{1}$ can be defined as $\mathrm{H}_{2}, \mathrm{C}_{2}$ as $\mathrm{CH}_{4}, \mathrm{C}_{3}$ as $\mathrm{C}_{2} \mathrm{H}_{6}, \mathrm{C}_{4}$ as $\mathrm{C}_{2} \mathrm{H}_{4}, \mathrm{C}_{5}$ as $\mathrm{C}_{2} \mathrm{H}_{2}$, and the involved nine fault types $\mathrm{U}_{1} \sim \mathrm{U}_{9}$ in this paper refer to the transformer normal circumstance, the transformer low temperature overheat fault, the transformer high temperature overheat fault, the transformer low energy discharge fault, the transformer high energy discharge fault, the transformer tree discharge fault, the transformer interturn discharge fault, the transformer core ground fault and the transformer tap switch fault.

To verify the feasibility and effectiveness of the transformer fault diagnosis method based on improved extension on intervals, 30 pairs of fault data of each fault type has been selected out to go through a diagnosis test. What's more, a benchmark processing has also been done when dealing with each pair of data. To prove the high efficiency of the transformer fault diagnosis method based on improved extension on intervals, the author of this paper has made a comparative analysis between the two pairs of diagnosis data: one is obtained through the discussed method in this paper, while another is obtained through the fuzzy clustering analytical method (FCAM) adopted in document ${ }^{[3]}$. And the comparative result of data test is shown as Table 2. 
Tab.2 Comparative Result of Data Test

\begin{tabular}{|c|c|c|c|c|c|}
\hline \multirow{2}{*}{ Fault Type } & \multirow{2}{*}{ Test Sample Amount } & \multicolumn{2}{|c|}{ The Correctly Recognized Fault Amount } & \multicolumn{2}{|r|}{ Recognition Rate } \\
\hline & & FCAM & Interval Extension Method & FCAM & Interval Extension Method \\
\hline $\mathrm{U}_{1}$ & 30 & 27 & 28 & $90 \%$ & $93 \%$ \\
\hline $\mathrm{U}_{2}$ & 30 & 28 & 29 & $93 \%$ & $97 \%$ \\
\hline $\mathrm{U}_{3}$ & 30 & 28 & 28 & $93 \%$ & $93 \%$ \\
\hline $\mathrm{U}_{4}$ & 30 & 26 & 27 & $87 \%$ & $90 \%$ \\
\hline $\mathrm{U}_{5}$ & 30 & 28 & 29 & $93 \%$ & $97 \%$ \\
\hline $\mathrm{U}_{6}$ & 30 & 25 & 28 & $83 \%$ & $93 \%$ \\
\hline $\mathrm{U}_{7}$ & 30 & 22 & 27 & $73 \%$ & $90 \%$ \\
\hline $\mathrm{U}_{8}$ & 30 & 26 & 28 & $87 \%$ & $93 \%$ \\
\hline $\mathrm{U}_{9}$ & 30 & 25 & 29 & $83 \%$ & $97 \%$ \\
\hline In Total & 270 & 235 & 253 & $87 \%$ & $93 \%$ \\
\hline
\end{tabular}

From Table 2, one can tell that the accuracy of the transformer fault diagnosis and judgment has been enhancedthrough the interval extension method. In addition, by comparing the run time of MATLAB program calculation in the case of FCAM and the Interval Extension Method in which it takes 220 seconds for FCAM to work out 270 pairs of data while it costs only 23 seconds by the adoption of the Interval Extension Method thanks to its leave-out of iterative computations, the conclusion can be drawn that the Interval Extension Method has greatly increased the effectiveness of transformer fault diagnosis, and can be regarded as a good way to solve the upsetting time-consuming problem in the engineering practical application because of the large data amount.

\section{Conclusion}

The oil-immersed transformer fault diagnosis method based on improved extension on intervals in this paper is to diagnose the typical transformer faults through the combination of interval extension method and improved analytic hierarchy process. By analyzing the fault characteristic quantities of typical gases under different transformer fault types and taking the leading features as the characteristic quantity in the transformer fault diagnosis, the author of this paper has made full use of the interval extension set to avoid the common problem of the traditional fault diagnosis method in which the calculation effectiveness is quite low because of the iterative computations. As is verified by the data test result, the method put forward in this paper can increase both the accuracy and the efficiency of transformer fault diagnosis.

\section{References}

[1] Li Jian, Research on Inner Fault Diagnosis Mode of Large-scale Power Transformer with Gases Dissolved in Oil as Characteristic Quantity [D], Chongqing University, 2001.

[2] Li Min, Xie Jun, Wang Yongqiang \& Lv Fangcheng, Transformer Fault Diagnosis Method Based on Improved FCAM of Quantum Particle Swarm Optimization [J], Electrical Logging and Instruments, 2015,v.52;No.61615:19-25. 
[3] Chen Tuo, Research on FCAM and Its Application in Power Transformer Fault Diagnosis [D], Xi'an University of Technology, 2008.

[4] Fan Junhui, Peng Daogang, Huang Yichao \& Yang Xuhong, Transformer Fault Diagnosis Method Based on Improved PSO and PNN, Measurement and Control Technology, 2016,v.35;No.28903:42-45+49. 